\title{
Aneurysmal Subarachnoid Hemorrhage in a 68-Year-old Hyperglycemic Female Patient: Case Report and Literature Review.
}

Paul Marcel Morgan. ${ }^{1}$

\begin{abstract}
Background: While hyperglycemia is intimately associated with uncontrolled diabetes mellitus (DM), recent clinical studies have demonstrated that hyperglycemia is also present in the early acute phase of stroke and is associated with poor prognosis and increased long-term mortality. About half of patients with acute hemorrhagic stroke also present with hyperglycemia upon admission. But more than $50 \%$ of patients with acute hemorrhagic stroke develop hyperglycemia even without a previous history of DM. This sheds new light on the relationship between DM, hyperglycemia, and hemorrhagic stroke, with a pathophysiology that is perhaps more profound than is conventionally understood. The Case: We report a case of a 68-year-old female, with a history of DM Type 2 and stage 3 hypertension who presents to the emergency room (ER) at the Western Regional Hospital in Belmopan City, Belize, with hemorrhagic stroke and hyperglycemia. Diffuse subarachnoid hemorrhage was found in the frontal, temporal, and parietal regions. Mild intraventricular hemorrhage was also observed in the frontal horns and basal cisterns. And small areas of intraparenchymal hemorrhage were present in the frontal lobes. The patient was stabilized and treated conservatively with calcium channel blockers, and diuretics. Conclusion: Despite a unifying consensus that is still pending, maintaining glucose levels between $110-120 \mathrm{mg} / \mathrm{dl}$ by using continuous insulin infusions after traumatic brain injury or aneurysmal subarachnoid hemorrhage may carry some clinical benefit with slightly improved outcome.
\end{abstract}

Key Words: Intracranial Hemorrhages, Traumatic Brain Injury, Intracranial Aneurysm, Hyperglycemia, Diabetes Mellitus (Source: MeSH-NLM).

\section{Introduction}

Non-traumatic brain injury resulting in hemorrhagic stroke is a debilitating condition and one of the major complications of uncontrolled hypertension. ${ }^{1}$ Despite hemorrhagic stroke being responsible for only $10 \%$ of all strokes, it is associated with a high morbidity and mortality index and is therefore a serious public health issue worldwide. ${ }^{1,}{ }^{2}$ However, diabetes often coexists with hypertension and more importantly both conditions are conventional risk factors for stroke. 3

While hyperglycemia is intimately associated with uncontrolled diabetes mellitus (DM), recent clinical studies have demonstrated that hyperglycemia is also present in the early acute phase of stroke and is associated with poor prognosis and increased long-term mortality. ${ }^{2}$ Kallikrein, a serine protease, has been recently elucidated as a crucial intermediate between hyperglycemia and profuse intracranial hemorrhage (ICH).

Kallikrein has since shed new light on the pathophysiology of hemorrhagic stroke. 4 Herein, we explore the relationship between DM and hyperglycemia and their roles in $\mathrm{ICH}$. However, the clinical benefit of lowering glucose levels in ICH still remains an active subject of debate and clinical inquiry. ${ }^{2}$ Increased efforts geared towards a consensus on hyperglycemia and hemorrhagic stroke is therefore imminent and necessary for optimal management of diabetic patients who present with profuse ICH.

\section{The Case}

A 68-year-old female patient with a history of stage 3 hypertension and inadequately controlled DM Type 2 presented to the Department of Accident and Emergency at the Western Regional Hospital in Belmopan
City, Belize. The patient ailed from consciousness disturbance, poor appetite, cephalgia, emesis, dehydration, and bilateral paresis. Symptoms were most pronounced within 12 hours before admission. on admission the patient had disturbed consciousness and her Glasgow coma score was $\mathrm{E}_{5} \mathrm{~V}_{3} \mathrm{M}_{2}$ (10). Vital signs in the $E R$ revealed a temperature of $36{ }^{\circ} \mathrm{C}$, pulse rate of 65 , respiratory rate of 16 , oxygen saturation $95 \%$, and a blood pressure of $190 / 110$. Fluid resuscitation was initiated and blood samples were taken.

ER workup revealed normal hemoglobin $13.5 \mathrm{~g} / \mathrm{dl}$ (norm. 13-18 g/dl), slightly decreased haemotocrit $39 \%$ (norm. 40-54\%) and mean corpuscular volume (MCV) $81 \mathrm{fl}$ (norm. 80-94 fl) without increased white blood cells (WBC). Differential WBC count revealed elevated neutrophils 87.3 (norm. 40-75 \%) and an elevated Erythrocyte Sedimentation Rate (ESR) of 67 (norm. M 0-10/ F 0-22 mm/hr). Fasting glucose level was elevated $(161 \mathrm{mg} / \mathrm{dl})$, while the urine analysis was unremarkable. A Computerized Axial Tomography (CAT) scan was performed using a multidetector scanner Brivo CT325. Continuous helical slices of $5 \mathrm{~mm}$ thick sections across the entire neurocranium were performed. Reconstruction at $3 \mathrm{~mm}$ slices and $1.5 \mathrm{~mm}$ intervals were also obtained. The osseous tissues and pneumotized cavities were studied using the bone window (Figure 1).

Diffuse subarachnoid hemorrhage was found in the frontal, temporal, and parietal regions. Mild intraventricular hemorrhage was also observed in the frontal horns and basal cisterns. Small areas of intraparenchymal hemorrhage were also present in the frontal lobes. General effacement of the cerebral gyri was noted upon observation. The cerebellar hemispheres, vermis, brainstem, and cerebello-pontine appeared normal in density and contour. Acceptable delimitation was observed between the grey and white matter. There were no hyperdense or hypodense spaces between the lesions (Figure 2).

1 School of Medicine for International Students, I-Shou University, Kaohsiung, Taiwan.

2 Department of Accident and Emergency, Western Regional Hospital, Belmopan City, Belize.

About the Author: Paul Morgan is currently a final year medical student in the four-year Doctor of Medicine (M.D.) Program at 1-Shou University School of Medicine for International Students. Paul is also an adjunct instructor of biochemistry at the University of Belize. 
Figure 1. Non-contrast CAT scan of the entire neurocranium with $5 \mathrm{~mm}$ continuous helical slices and $3 \mathrm{~mm}$ slice reconstruction at 1.5 .

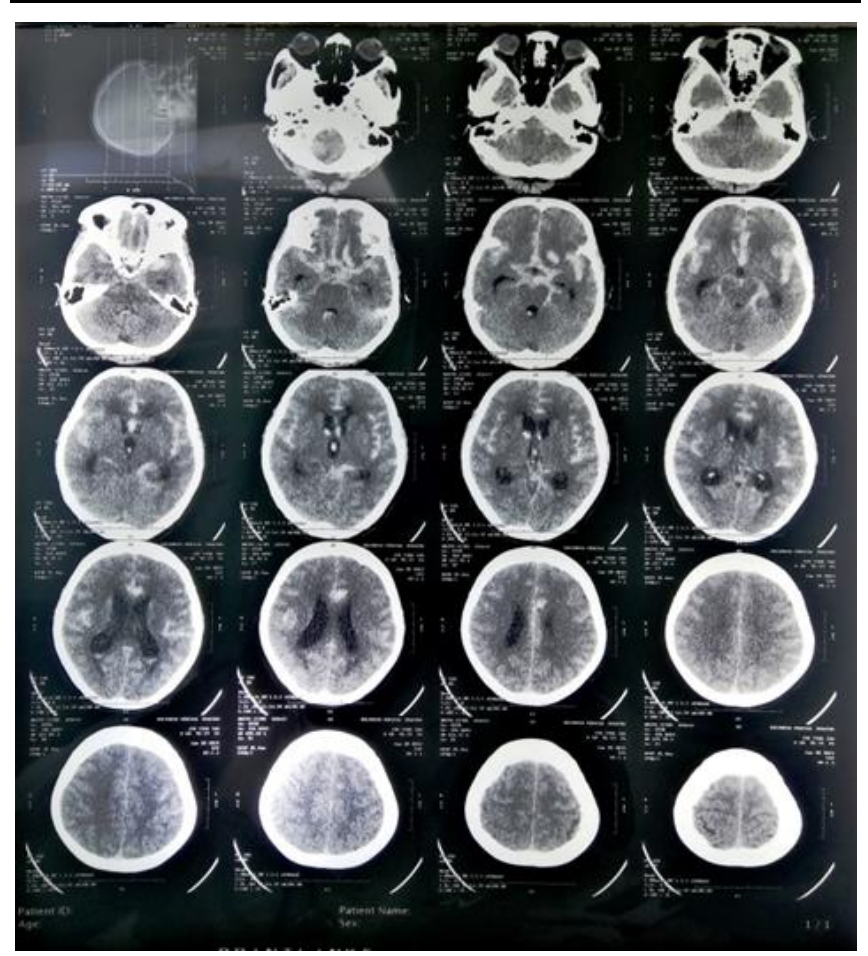

The patient's fluids were maintained with $500 \mathrm{cc}$ of Ringer's lactate solution and treatment was conservative with $90 \mathrm{mg}$ ER PO QD nifedipine, $200 \mathrm{mg} / \mathrm{kg}$ IV infused mannitol to reduce intracranial pressure associated with brain edema. A central line was established

Figure 2. Axial non-contrast CAT image showing diffuse subarachnoid hemorrhage, intraparenchymal hemorrhage, and mild intraventricular hemorrhage.

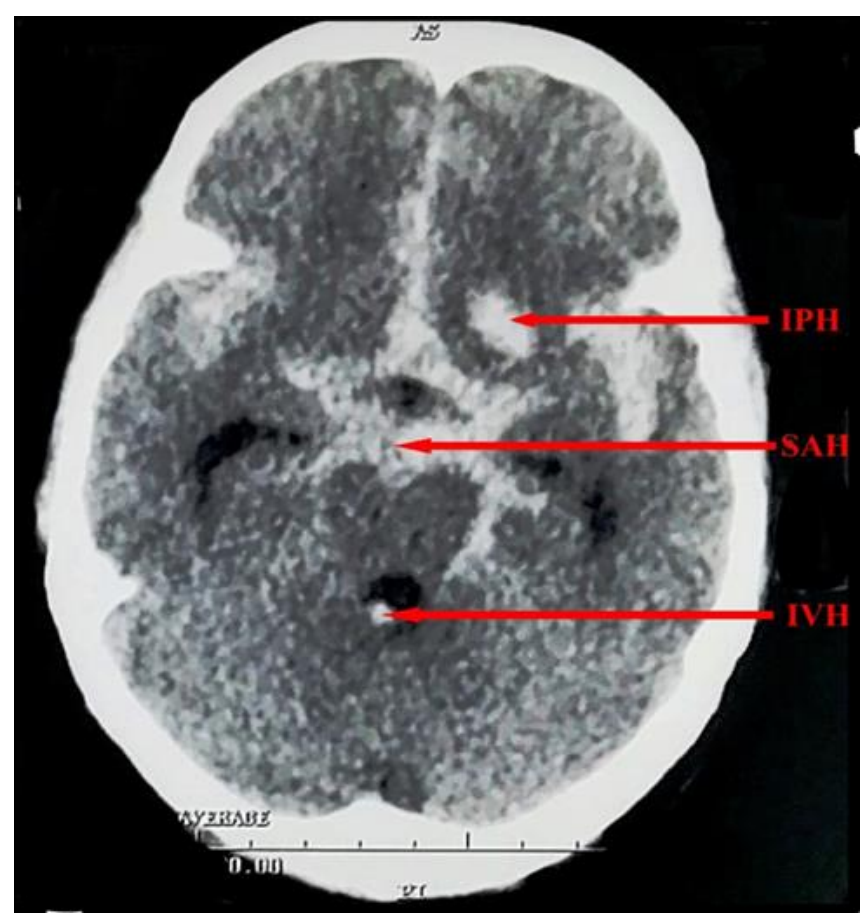

via the internal jugular vein followed by rapid sequence intubation (RSI). Despite burr-hole evacuation being the optimal choice of intervention, there are currently no neurosurgical facilities at the treatment hospital to facilitate the lifesaving intervention. Our patient was transferred to the largest equipped referral trauma center in Belize City, Karl Kushner Memorial Hospital (KHMH) for further expert intervention.

\section{Discussion}

The Bondage of DM, Hemorrhagic Stroke, and Hyperglycemia

Intracranial aneurysms (IAS) occur in about $5 \%$ of the general population and are characterized by localized dilatation of cerebral arteries with a high predisposition to rupture5. Ruptured IAs are the leading cause of hemorrhagic stroke, and are consequently responsible for $85 \%$ of subarachnoid hemorrhages (Figure 3 ). ${ }^{6}$

While the pathophysiology of ischemic stroke and hemorrhagic stroke differ, hypertension, either alone or concomitant with other etiological factors is overwhelmingly the primary culprit in cases of stroke. ${ }^{3}$ DM being a premorbid requisite for hypertension, few studies have explored the intricacy between hyperglycemia and hemorrhagic stroke. Diabetes alone quadruples the risk of intracranial hemorrhage. Several clinical studies have revealed that diabetes and hyperglycemia are associated with poor clinical outcomes flowing from ICH.1, 2

Nearly $50 \%$ of all acute stroke patients present with hyperglycemia upon admission as in the case of our patient.2, 8, 9 Despite this fact, the effects of elevated glucose in a stroke patient, with or without a history of DM, and the role of DM in ICH is not fully understood.1, 3 However, patterns of association between DM and ICH are plausible. Thus, there may be several mediating mechanisms by which hyperglycemia contributes to small vessel disease and ICH volume expansion. ${ }^{10}$ How DM contributes to a hyperglycemic state after aneurysm rupture is therefore worth investigating.

\section{Hemorrhagic Stroke and TBI: Smoking Gun Parallels}

Traumatic Brain Injury (TBI) is classified into two main categories (i.e. primary and secondary).9, 11-15 Primary injuries occur as a consequence of shearing and compression due to an applied physical force. ${ }^{14}$ Secondary injury succeeds primary injury, initiates within a few hours, and terminates within a few days.4, 10, 12, 14, 16 Secondary injury is rather complex and triggers cranial and systemic complications which are both notoriously associated with poor clinical outcomes.4, 10, 12, 14, 16 Intracranial hypertension, cerebral edema, calcium ion toxicity, infection, and vasospasm are considered to be cranial complications while systemic complications encompass hypoxemia, hypotension, hypertension, hypoglycemia and hyperglycemia.4, 10, 12, 14, 16 Although ischemic stroke, hemorrhagic stroke, subarachnoid hemorrhage, and intraparenchymal hemorrhage are mechanistically non-traumatic in nature, they all elicit the same hemostatic responses with cranial and systemic complications. Shi et al. reports that TBI with DM Type 2 has a much higher mortality (14\%) than TBI without DM Type II (8.2\%). 1, 10, $14,15,17$ And DM Type 1 has a higher (17.1\%) mortality rate than DM Type 2. It suggests that DM has the potential to be an independent predictor of poor outcome as insulin deficiency, a direct correlation to elevate glucose levels, seems to be a major contributor to increased mortality after TBI or stroke.1, 15, 18 If so, then such a clinical pattern poses another important query; is there a glucose threshold with respect to persistent hyperglycemia?

\section{The Hyperglycemic State}

Persistent hyperglycemia can be defined as average daily blood glucose levels in excess of $149 \mathrm{mg} / \mathrm{dl}$ for the first week following TBI or stroke - cerebral vascular insult. ${ }^{13}$ A cohort study of 834 patients found that blood glucose below $145 \mathrm{mg} / \mathrm{dl}$ significantly reduces lactate production, and results in optimal lactate/glucose and oxygen/glucose indices following a cerebral vascular insult. ${ }^{13}$ However, the pathophysiological 
and bimolecular mechanism by which aberrant glucose concentrations worsens clinical outcomes is still a subject of on-going research.9, 13-15

The hyperglycemic state is a consequence of a systemic inflammatory response syndrome (SIRS), which is a cascade of local deleterious mechanisms involving increase in oxidative stress, recruitment of multiple inflammatory cytokines, TNF- $\alpha, \mathrm{IL}-6, \mathrm{CD} 11 \mathrm{~d}$, and an induction of excitotoxicity. ${ }^{11,} 19$ Studies demonstrate that TNF- $\alpha$ regulates the role and quantity of glucose by elevating levels or pre-adipocyte genes and decreasing adipocyte specific genes ultimately resulting in hyperglycemia. ${ }^{15}$ The inflammatory response cascade also contributes to hyperglycemia by increasing the level of corticotrophin-releasing hormone (CRH) and stimulating the release of adrenocorticotropic hormone (ACTH) from the anterior pituitary. ${ }^{15}$ Not surprisingly, our patient's ESR was markedly elevated, greater than 3 times above the normal range indicating active inflammation. Nevertheless, the complete relationship between SIRS and hyperglycemia needs to be further developed as well. 15,19

\section{A Novel Antiplatelet Protein}

A 2017 study investigated the effect of diabetes and hyperglycemia on the mechanism of acute hematoma expansion. ${ }^{20}$ Their studies revealed that hyperglycemia increases hematoma formation and the mechanism is regulated by an osmotic-sensitive serine protease, kallikrein, which inhibits platelet aggregation.4, 20 The authors further suggest that plasma kallikrein is activated by coagulation factor XII via a contact activation system which is crucial to the intrinsic coagulation cascade and innate inflammation.20

Plasma kallikrein is well known for its role in the proteolytic cleavage of kininogen which yields bradykinin leading to vascular dilation and increased permeability through bradykinin receptors 1 and 2.4 Feener et al. results suggest that the increase in hematoma expansion associated with hyperglycemia is arbitrated by an associated increase in osmolality, which is consistent with multiple reports postulating that raised plasma osmolality on admission after acute hemorrhagic stroke increases the risk of mortality.4, 7, 18, 20 Interestingly, the study also demonstrated that hyperosmolar mannitol and hyperosmolar salt activated plasma kallikrien in the same manner by which elevated glucose concentrations do.

Thus, hyperglycemia increases hematoma expansion in $\mathrm{ICH}$ and the exacerbation of hematoma expansion is mediated by kallikrein. Plasma kallikrein is therefore a potential target for controlling hematoma expansion in the setting of hyperglycemia. Currently, there are no FDA approved small molecule inhibitors for kallikrein.21, 22 As such, small molecule inhibitors that can effectively mediate TNF- $\alpha$ and kallikrein levels immediately after hemorrhagic stroke may reduce cerebral bleeding, control glucose levels and significantly improve recovery, which may lead to better clinical outcomes particularly in diabetics.

\section{Tight Glucose Control with Insulin Infusion}

To determine whether elevated blood glucose levels cause or exacerbate secondary brain injury following TBI, Meng et al. conducted a prospective study of 240 adults. ${ }^{2,} 11$ Patients with severe TBI were randomly assigned to either of two groups; a tightly regulated glucose group and a control group. ${ }^{11}$ The glucose levels for the tightly regulated group were maintained at $80 \mathrm{mg} / \mathrm{dl}$ using continuous insulin infusion therapy throughout their stay at the hospital while the control group adhered to a conventional glucose control where insulin was given only if glucose levels exceeded $200 \mathrm{mg} / \mathrm{dl}^{11}$ The results of the study revealed that significantly more patients in the intensive insulin therapy group had good outcomes - Glasgow Outcome Scale (COS) between 4 and 5 versus that of the conventional therapy group. Interestingly, insulin therapy was also associated with significantly lower infection rates and shorter ICU stay. ${ }^{11}$ The six month mortality outcomes in both groups were, however, similar. Despite these findings, many other clinical studies of patients with hemorrhagic stroke and TBI have found little benefit from tight glucose regulation and many propose concerns about hypoglycemia and further neuronal injury.3, 8, 11, 13, 15, 19 In conclusion, despite the discrepancies in a unifying consensus, maintaining glucose levels between $110-120 \mathrm{mg} / \mathrm{dl}$ by using continuous insulin infusions after TBI or hemorrhagic stroke does carry some clinical benefit with slightly improved outcomes. 3, 8, 11, 13-15, 19 
Figure 3. Hypothetical pathways which explore the relationship between DM, hyperglycemia, and ICH. Uncontrolled DM contributes to a persistent hyperglycemic state. Hemorrhagic stroke also contributes to a hyperglycemic state. DM is also a major risk factor for hypertension. Uncontrolled hypertension can be equated to an acute force like that of TBI leading to cerebral insult. Hyperglycemia leads to increased osmolality which in turn up-regulates and activates plasma kallikrein. ${ }^{20}$ Hyperglycemia also triggers SIRS which up-regulates TNF- $\alpha$ and, elevated TNF- $\alpha$ also contributes positively to the hyperglycemic state. Additionally, infection also has a positive feedback on the cytokine TNF- $\alpha .4,14$

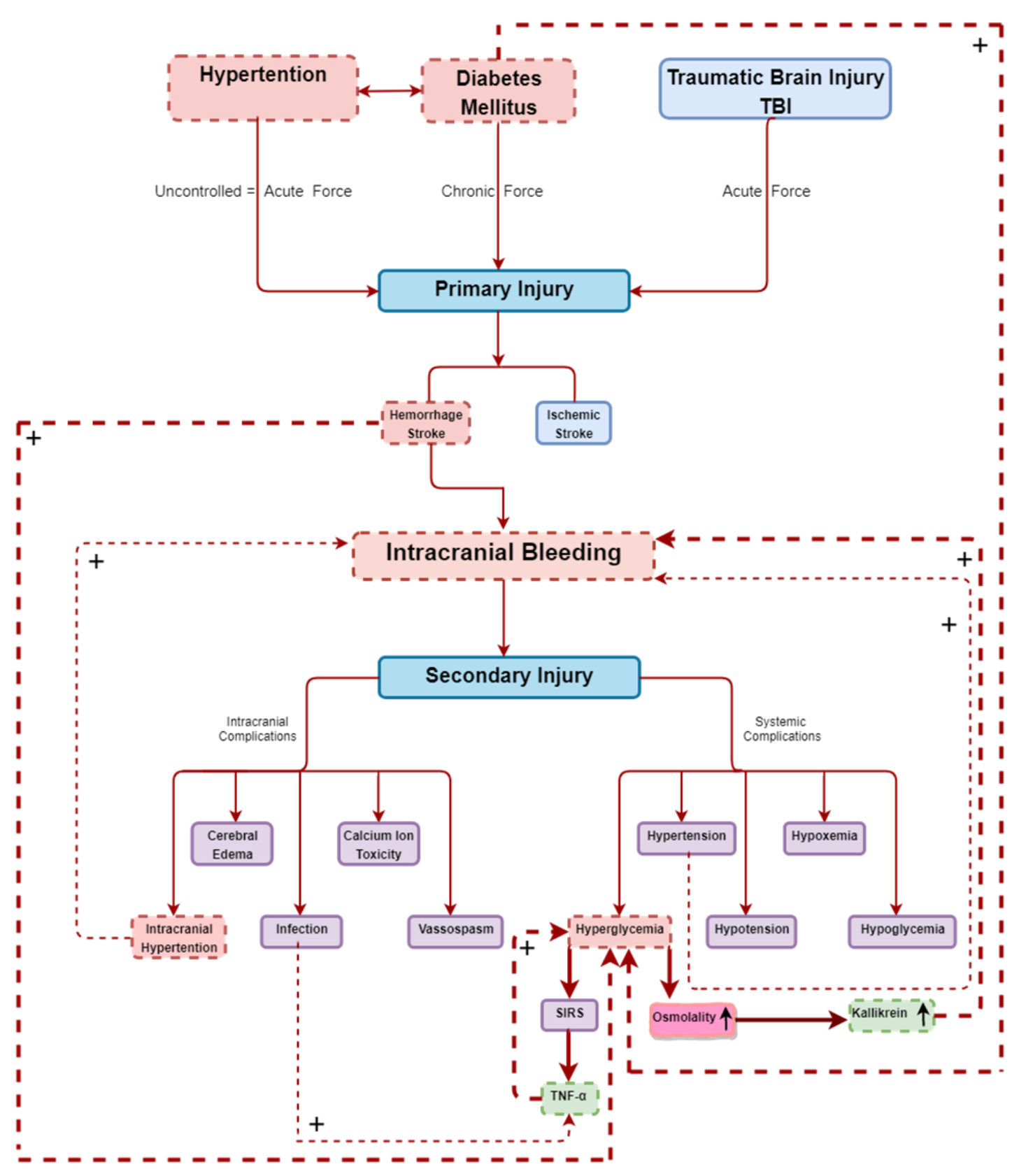




\section{References}

1. Hesami O, Kasmaei HD, Matini F, Assarzadegan F, Mansouri B, Jabbehdari S. Relationship between intracerebral hemorrhage and diabetes mellitus: a casecontrol study. J Clin Diagn Res. 2015 Apr;9(4):0C08-10

2. Chen R, Ovbiagele B, Feng W. Diabetes and Stroke: Epidemiology, Pathophysiology, Pharmaceuticals and Outcomes. Am J Med Sci. 2016 Apr;351(4):380-6.

3. Hyvärinen M, Tuomilehto J, Mähönen $M$, Stehouwer Coen DA, Pyörälä K, Zethelius $B$, et al. Hyperglycemia and Incidence of Ischemic and Hemorrhagic StrokeComparison Between Fasting and 2-Hour Clucose Criteria. Stroke. 2009 May;40(5):1633-7.

4. Simão F, Feener EP. The Effects of the Contact Activation System on Hemorrhage. Front Med (Lausanne). 2017 Jul 31;4:121.

5. Chalouhi N, Hoh Brian L, Hasan D. Review of Cerebral Aneurysm Formation, Growth, and Rupture. Stroke. 2013 Dec;44(12):3613-22.

6. Fennell VS, Kalani MYS, Atwal C, Martirosyan NL, Spetzler RF. Biology of Saccular Cerebral Aneurysms: A Review of Current Understanding and Future Directions. Front Surg. 2016 Jul 25;3:43.

7. Alvis-Miranda H, Alcalá-Cerra G, Moscote-Salazar L. Glycemia in Spontaneous Intracerebral Hemorrhage: Clinical Implications. Romanian Neurosurgery. 2014;21(3):315-26.

8. Marion DW. Optimum serum glucose levels for patients with severe traumatic brain injury. F1000 Med Rep. 2009; 1: 42.

9. Myers RB, Lazaridis C, Jermaine CM, Robertson CS, Rusin CG. Predicting Intracranial Pressure and Brain Tissue Oxygen Crises in Patients With Severe Traumatic Brain Injury. Crit Care Med. 2016 Sep;44(9):1754-61.

10. Boulanger M, Poon MTC, Wild SH, Al-Shahi Salman R. Association between diabetes mellitus and the occurrence and outcome of intracerebral hemorrhage. Neurology. 2016 Aug 30;87(9):870-8.

11. Yang $M$, Guo $Q$, Zhang $X$, Sun S, Wang $Y$, Zhao L, et al. Intensive insulin therapy on infection rate, days in NICU, in-hospital mortality and neurological outcome in severe traumatic brain injury patients: A randomized controlled trial. Int J Nurs Stud. 2009 Jun;46(6):753-8.

12. Chiu C-C, Liao $Y-E$, Yang L-Y, Wang J-Y, Tweedie D, Karnati HK, et al. Neuroinflammation in animal models of traumatic brain injury. I Neurosci Methods. 2016 Oct 15;272:38-49.
13. Salim A HP, Dubose J, Brown C, Inaba K, Chan LS, Margulies D. Persisten hyperglycemia in severe traumatic brain injury: an independent predictor of outcome. Am Surg. 2009 Jan;75(1):25-9.

14. Shi J, Dong B, Mao Y, Guan W, Cao J, Zhu R, et al. Review: Traumatic brain injury and hyperglycemia, a potentially modifiable risk factor. Oncotarget. 2016 Oct $25 ; 7(43): 71052-71061$

15. Bosarge PL, Shoultz TH, Griffin RL, Kerby JD. Stress-induced hyperglycemia is associated with higher mortality in severe traumatic brain injury. J Trauma Acute Care Surg. 2015 Aug;79(2):289-94.

16. Siesjö BK. Basic mechanisms of traumatic brain damage. Annals of Emergency Medicine. Ann Emerg Med. 1993 Jun;22(6):959-69.

17. Muñoz-Rivas N, Méndez-Bailón M, Hernández-Barrera V, de Miguel-Yanes JM, Jimenez-Carcia R, Esteban-Hernández J, et al. Type 2 Diabetes and Hemorrhagic Stroke: A Population-Based Study in Spain from 2003 to 2012. J Stroke Cerebrovasc Dis. 2016 Jun;25(6):1431-43.

18. Owolabi L, Nagode M, Ibrahim A, Uloko A, Gezawa I, Datti M. Stroke in patients with diabetes mellitus: a study from North Western Nigeria. Afr Health Sci. 2016 Sep;16(3):781-789.

19. Hernando A-M, Cabriel A-C, Luis Rafael M-S. Clycemia in Spontaneous Intracerebral Hemorrhage: Clinical Implications. Romanian Neurosurgery. 2014; 21(3): 315-26.

20. Simão F, Ustunkaya T, Clermont AC, Feener EP. Plasma kallikrein mediates brain hemorrhage and edema caused by tissue plasminogen activator therapy in mice after stroke. Blood. 2017 Apr 20;129(16):2280-2290.

21. Paul M. Morgan MM, Velabo Mdluli. Fragment-Based Design of a Potential TNF$\alpha$ Inhibitor Inspired by Castanospermine and Methyl Phenylacetate. E-Da Medical Journal. 2018; 5(3): 9-18.

22. Paul Morgan MM, Chih-Wen Lin. In silico Study of 1,687 FDA Approved Drugs and 612 Natural Products Reveals Benzydamine's Potential as a Direct Inhibitor of TNF- $\alpha$. IRJPMS. 2018; 2(2): 13-8.

23. Meier R, Béchir M, Ludwig S, Sommerfeld J, Keel M, Steiger $P$, et al. Differential temporal profile of lowered blood glucose levels $(3.5$ to $6.5 \mathrm{mmol} / \mathrm{l}$ versus 5 to $8 \mathrm{mmol} / \mathrm{l}$ ) in patients with severe traumatic brain injury. Crit Care. $2008 ; 12(4): R 98$

\section{Acknowledgments}

The author thanks Taiwan ICDF and I-Shou University School of Medicine for International Students, Taiwan, for providing the learning environment conducive to development as physician scientists. The authors also thank Paul Morgan Sr. for editing the manuscript.

\section{Conflict of Interest Statement at Funding}

The Authors have no funding, financial relationships or conflicts of interest to disclose.

\section{Author Contributions}

Conceptualization, Software, Formal Analysis, Writing - Original Draft, Writing - Review \& Editing, Project Administration: P.M.M.

Cite as:

Morgan PM. Aneurysmal Subarachnoid Hemorrhage in a 68-Year-old Hyperglycemic Female Patient: Case Report and Literature Review. Int J Med Students. 2019 MayAug; $7(2): 45-49$. 